\title{
Biased assimilation, homophily, and the dynamics of polarization
}

\author{
Pranav Dandekar ${ }^{a, 1}$, Ashish Goel ${ }^{b}$, and David T. Lee \\ Departments of a Management Science and Engineering, ${ }^{b}$ Management Science and Engineering and (by courtesy) Computer Science, and ${ }^{c}$ Electrical \\ Engineering, Stanford University, Stanford CA 94305
}

Edited by Jon Kleinberg, Cornell University, Ithaca, NY, and approved February 28, 2013 (received for review October 3, 2012)

We study the issue of polarization in society through a model of opinion formation. We say an opinion formation process is polarizing if it results in increased divergence of opinions. Empirical studies have shown that homophily, i.e., greater interaction between like-minded individuals, results in polarization. However, we show that DeGroot's well-known model of opinion formation based on repeated averaging can never be polarizing, even if individuals are arbitrarily homophilous. We generalize DeGroot's model to account for a phenomenon well known in social psychology as biased assimilation: When presented with mixed or inconclusive evidence on a complex issue, individuals draw undue support for their initial position, thereby arriving at a more extreme opinion. We show that in a simple model of homophilous networks, our biased opinion formation process results in polarization if individuals are sufficiently biased. In other words, homophily alone, without biased assimilation, is not sufficient to polarize society. Quite interestingly, biased assimilation also provides a framework to analyze the polarizing effect of Internet-based recommender systems that show us personalized content.

$T^{\mathrm{T}}$ he issue of polarization in society has been extensively studied and vigorously debated in the academic literature as well as the popular press over the last few decades. In particular, are we as a society getting more polarized? If so, why, and how can we fix it? Different empirical studies arrive at different answers to this question depending on the context and the metric used to measure polarization.

Evidence of polarization in politics has been found in the increasingly partisan voting patterns of the members of Congress $(1,2)$ and in the extreme policies adopted by candidates for political office (3). McCarty et al. (4) claim via rigorous analysis that America is polarized in terms of political attitudes and beliefs. Phenomena such as segregation in urban residential neighborhoods (5-7), the rising popularity of overtly partisan television news networks $(8,9)$, and the readership and linking patterns of blogs along partisan lines (10-13) can all be viewed as further evidence of polarization. On the other hand, it has also been argued on the basis of detailed surveys of public opinion that society as a whole is not polarized, even though the media and the politicians make it seem so $(14,15)$. We adopt the view that polarization is not a property of a state of society; instead it is a property of the dynamics through which individuals form opinions. We say that opinion formation dynamics are polarizing if they result in an increased divergence of opinions.

It has been argued using empirical studies that homophily, i.e., greater interaction between like-minded individuals, results in polarization $(12,16,17)$. This argument has been used to claim that the rise of cable news, talk radio, and the Internet has contributed to polarization: the increased diversity of information sources coupled with the increased ability to narrowly tailor them to one's specific tastes (either manually or algorithmically through, for example, recommender systems) has an echo-chamber effect that ultimately results in increased polarization.

A rich body of work attempts to explain polarization through variants of a well-known mathematical model of opinion formation proposed by DeGroot (18). In DeGroot's model, individuals are connected to each other in a social network. The edges of the network have associated weights representing the extent to which neighbors influence each other's opinions. Individuals update their opinion as a weighted average of their current opinion and that of their neighbors. Variants of this model (e.g., refs. 19-22) explain the empirically observed persistent disagreement on many issues by, for example, introducing stubborn individuals (i.e., individuals with unchanging opinions) into the original model. However, we show that repeated averaging of opinions, which underlies these models, always results in opinions that are less divergent compared with the initial opinions, even if individuals are arbitrarily homophilous. As a result, this entire body of work appears to fall short of explaining polarization which is generally perceived to mean an increased divergence of opinions, not just persistent disagreement. In this paper, we seek a more satisfactory model of opinion formation that $(a)$ is informed by a theory of how individuals actually form opinions and $(b)$ produces an increased divergence of opinions under intuitive conditions.

We base our model on a well-known phenomenon in social psychology called biased assimilation, according to which individuals process new information in a biased manner whereby they readily accept confirming evidence while critically examining disconfirming evidence. Suppose that individuals with opposing views on an issue are shown mixed or inconclusive evidence. Intuitively, exposure to such evidence would engender greater agreement, or at least a moderation of views. However, in a seminal paper, Lord et al. (23) showed that biased assimilation causes individuals to arrive at more extreme opinions after being exposed to identical, inconclusive evidence. This finding has been reproduced in many different settings over the years (e.g., refs. 24-26). We use biased assimilation as the basis of our model of opinion formation and show that in our model homophily alone, without biased assimilation, is not sufficient to polarize society.

It has been argued (27) that biased assimilation can be countered by surprising validators: Individuals are more likely to carefully consider disconfirming evidence if it is presented by a source that is otherwise similar to them. Centola (28) empirically showed that individuals are much more likely to adopt health behaviors when they are a part of more homophilous networks. We show that a stylized model of surprising validators does indeed reduce polarization as we define it in this paper.

Finally, we analyze the polarizing effects of recommender systems that are widely used on the Internet to make personalized recommendations (e.g., search results, news articles, products) to individuals. We analyze three recommender algorithms-SimpleSALSA, SimplePPR, and SimpleICF - that are similar in spirit to commonly used recommender algorithms. For a simple, natural model of the underlying user-item graph, and under reasonable assumptions, we show that SimplePPR, which recommends the item that is most relevant to a user based on a PageRank-like (29) score, is always polarizing. On the other hand, SimpleSALSA and SimpleICF, which first choose a random item liked by the user

Author contributions: P.D., A.G., and D.T.L. designed research, performed research, contributed new reagents/analytic tools, analyzed data, and wrote the paper.

The authors declare no conflict of interest.

This article is a PNAS Direct Submission.

${ }^{1}$ To whom correspondence should be addressed. E-mail: ppd@stanford.edu.

This article contains supporting information online at www.pnas.org/lookup/suppl/doi:10. 1073/pnas.1217220110/-/DCSupplemental. 
and recommend an item similar to that item, are polarizing only if individuals are biased. Thus, biased assimilation also provides a useful framework to understand whether recommender systems contribute to polarization.

\section{Model}

Our opinion formation process unfolds over a social network represented by a connected weighted undirected graph $G=(V, E, w)$. The nodes in $V$ represent individuals and the edges represent friendships or relationships between them. Let $|V|=n$. An edge $(i, j) \in E$ is associated with a weight $w_{i j}>0$ representing the degree of influence $i$ and $j$ have on each other. Each individual $i \in V$ also has an associated weight $w_{i i} \geq 0$ representing the degree to which the individual weights his own opinions. We denote by $N(i)$ the set of neighbors of $i$; that is, $N(i):=\{j \in V:(i, j) \in E\}$.

An individual $i$ has an opinion $x_{i}(t) \in[0,1]$ at time step $t=0,1$, $2 \ldots$. The extreme opinions 0 and 1 represent opposing points of view on an issue. So $x_{i}(t)$ can be interpreted as individual $i$ 's degree of support at time $t$ for the position represented by 1 and $1-x_{i}(t)$ as the degree of support for the position represented by 0 . Let $\mathbf{x}(t) \in[0,1]^{n}$ denote the vector of opinions at time $t$. An opinion formation process is a description of how individuals update their opinions; i.e., for each individual $i \in V$, it defines $x_{i}(t+1)$ as a function of the vector of opinions, $\mathbf{x}(t)$, at time $t$.

Measuring Polarization. We view polarization as a property of an opinion formation process instead of a property of a state of the network. We characterize polarization as a verb as opposed to a noun; i.e., we say that an opinion formation process is polarizing if it results in an increased divergence of opinions. One could mathematically capture divergence of opinions in many different ways. We measure divergence in terms of the network disagreement index defined below.

Definition 1. Network Disagreement Index. Given a graph $G=(V, E, w)$ and a vector of opinions $\mathbf{x} \in[0,1]^{n}$ of individuals in $V$, the network disagreement index $\eta(G, \mathbf{x})$ is defined as

$$
\eta(G, \mathbf{x}):=\sum_{(i, j) \in E} w_{i j}\left(x_{i}-x_{j}\right)^{2}
$$

Consider an opinion formation process over a network $G=(V$, $E, w)$ that transforms a set of initial opinions $\mathbf{x} \in[0,1]^{n}$ into a set of opinions $\mathbf{x}^{\prime} \in[0,1]^{n}$. Then, we say the process is polarizing if $\eta\left(G, \mathbf{x}^{\prime}\right)>\eta(G, \mathbf{x})$, and vice versa.

The network disagreement index (NDI) is similar to the notion of social cost used by Bindel et al. (22). Each term $w_{i j}\left(x_{i}-x_{j}\right)^{2}$ can be viewed as the cost of disagreement imposed upon $i$ and $j$. This view that the social cost depends on the magnitude of the difference of opinions along edges is consistent with theories in social psychology according to which attitude conflicts in relationships are a source of psychological stress or instability $(30,31)$. The NDI captures the phenomenon of issue radicalization, i.e., preexisting groups of individuals becoming progressively more extreme. Admittedly, it does not entirely capture an aspect of polarization called issue alignment (32) whereby individuals with diverse opinions organize into ideologically coherent, but opposing factions. However, there is significant empirical evidence $(4,27,32)$ that issue radicalization is more prevalent compared with issue alignment, and hence NDI captures the most salient aspects of polarization. Many of our results hold for more general measures of divergence, which we discuss in a later section.

DeGroot's Repeated Averaging Process. In his seminal work on opinion formation, DeGroot (18) proposed a process where at each time step, individuals simultaneously update their opinion to the weighted average of their neighbors' and their own opinion at the previous time step.
Definition 2. DeGroot's Repeated Averaging Process. The opinion of individual $i$ at time $t+1, x_{i}(t+1)$, is given by

$$
x_{i}(t+1)=\frac{w_{i i} x_{i}(t)+s_{i}(t)}{w_{i i}+d_{i}}
$$

where $s_{i}(t):=\sum_{j \in N(i)} w_{i j} x_{j}(t)$ is the weighted sum of the opinions of $i$ 's neighbors, and $d_{i}:=\sum_{j \in N(i)} w_{i j}$ is i's weighted degree.

Recall that $x_{j}(t)$ and $1-x_{j}(t)$ represent the degree of support for extremes 1 and 0 , respectively. Then, opinion update under DeGroot's process is equivalent to taking a weighted average of the total support for 0 and that for 1 . The weight that individual $i$ places on 1 (and on 0 ) is computed by summing the degrees of support of $i$ 's neighbors weighted by the influence of each neighbor on $i$.

Biased Opinion Formation Process. We generalize DeGroot's process to account for biased assimilation. Biased assimilation is a well-known phenomenon in social psychology described by Lord et al. (23, p. 2098) in their seminal paper as follows:

People who hold strong opinions on complex social issues are likely to examine relevant empirical evidence in a biased manner. They are apt to accept "confirming" evidence at face value while subjecting "disconfirming" evidence to critical evaluation, and as a result to draw undue support for their initial positions from mixed or random empirical findings.

Lord et al. (23) showed through experiments that biased assimilation of mixed or inconclusive evidence does indeed result in more extreme opinions.

To account for biased assimilation, we propose a biased opinion formation process. Recall that $x_{i}(t)$ can be viewed as the degree of support for the position represented by 1 . Individuals weight confirming evidence more heavily relative to disconfirming evidence by updating their opinions as follows: Individual $i$ weights each neighbor $j$ 's support for 1 [i.e., $x_{j}(t)$ ] by an additional factor $\left(x_{i}(t)\right)^{b_{i}}$, where $b_{i} \geq 0$ is a bias parameter. Therefore, $x_{i}(t+1) \propto$ $\left(x_{i}(t)\right)^{b_{i}} w_{i j} x_{j}(t)$. Similarly, $i$ weights $j$ 's support for 0 [i.e., $1-x_{j}(t)$ ] by $\left(1-x_{i}(t)\right)^{b_{i}}$, and so $\left(1-x_{i}(t+1)\right) \propto\left(1-x_{i}(t)\right)^{b_{i}} w_{i j}\left(1-x_{j}(t)\right)$. Informally, $b_{i}$ represents the bias with which $i$ assimilates his neighbors' opinions.

Illustrative Example. Consider a graph with two nodes, $i$ and $j$, connected by an edge with a weight $w_{i j}$. Then, according to the biased opinion formation process, $i$ 's opinion at time $t+1, x_{i}(t+1)$, is given by

$$
x_{i}(t+1)=\frac{w_{i i} x_{i}(t)+\left(x_{i}(t)\right)^{b_{i}} w_{i j} x_{j}(t)}{w_{i i}+\left(x_{i}(t)\right)^{b_{i}} w_{i j} x_{j}(t)+\left(1-x_{i}(t)\right)^{b_{i}} w_{i j}\left(1-x_{j}(t)\right)} .
$$

More generally, the opinion update of individual $i$ in the biased opinion formation process is defined as shown below.

Definition 3. Biased Opinion Formation Process. Under the biased opinion formation process, the opinion of individual $i$ at time $t+1$, $x_{i}(t+1)$, is given by

$$
x_{i}(t+1)=\frac{w_{i i} x_{i}(t)+\left(x_{i}(t)\right)^{b_{i}} s_{i}(t)}{w_{i i}+\left(x_{i}(t)\right)^{b_{i}} s_{i}(t)+\left(1-x_{i}(t)\right)^{b_{i}}\left(d_{i}-s_{i}(t)\right)},
$$

where, as before, $s_{i}(t):=\sum_{j \in N(i)} w_{i j} x_{j}(t)$ is the weighted sum of the opinions of $i$ 's neighbors, and $d_{i}:=\sum_{j \in N(i)} w_{i j}$ is $i$ 's weighted degree. Observe that when $b_{i}=0,[3]$ is identical to [2]; i.e., DeGroot's process is a special case of our process and corresponds to unbiased assimilation. More generally, biased assimilation can be modeled by making $i$ 's opinion update proportional to $\beta_{i}\left(x_{i}(t)\right)$ $s_{i}(t)$, where the bias function $\beta_{i}:[0,1] \rightarrow[0,1]$ is nondecreasing.

Connection with Urn Models. Urn models are an elegant abstraction and have been used to analyze the properties of a wide 
variety of probabilistic processes. DeGroot's process and our biased opinion formation process have the following analogous urn dynamics. Let $x_{i}(t)$ denote the fraction of RED balls in individual $i$ 's urn at time $t$ and $1-x_{i}(t)$ denote the corresponding fraction of BLUE balls:

- $\quad$ Step 1 (common): At each time step, $i$ chooses a neighbor $j$ with probability proportional to $w_{i j}$ and inspects a ball chosen uniformly at random from $j$ 's urn. Note that $i$ does not remove the ball from $j$ 's urn.

- $\quad$ Step 2 (DeGroot's process): $i$ adds a ball of the same color as the inspected ball to his urn and discards a ball chosen uniformly at random from his urn.

- $\quad$ Step 2 (Biased opinion formation process with $b_{i}=1$ ): $i$ also inspects a ball chosen uniformly at random from his own urn. If the colors of the two inspected balls match, $i$ adds a ball of the same color to his urn and discards a ball chosen uniformly at random from his urn.

Biased Assimilation by a Single Agent in a Fixed Environment. Here we demonstrate that our model of biased assimilation mathematically reproduces the empirical findings of Lord et al. (23). We analyze the change in opinion of a single individual as a function of his bias parameter when he is exposed to opinions from a fixed environment. The fixed environment represents sources of information that influence the individual's opinion, but can be assumed to remain unaffected by the individual's opinion, such as the news media, the Internet, the organizations that the individual is a part of, etc.

For this section, we denote by $x(t) \in[0,1]$ the individual's opinion at time $t$ and by $b \geq 0$ the individual's bias parameter. Let the individual's weight on his own opinion be $w_{i i}=w$. Let $s \in(0,1)$ denote the (time-invariant) weighted average of the opinions of all sources in the individual's environment. Then, from [3], the individual's opinion at time $t+1$ is given by

$$
x(t+1)=\frac{w x(t)+(x(t))^{b} s}{w+(x(t))^{b} s+(1-x(t))^{b}(1-s)} .
$$

Given $s \in(0,1)$, and $b \neq 1$, we define

$$
\hat{x}(s, b):=\frac{s^{1 /(1-b)}}{s^{1 /(1-b)}+(1-s)^{1 /(1-b)}}
$$

as the polarization threshold for the individual. We show that when the individual is sufficiently biased (i.e., $b>1$ ), the polarization threshold $\hat{x}$ is an unstable equilibrium; i.e., in equilibrium the individual's opinion goes to 1 or 0 depending on whether the initial opinion was greater than or less than $\hat{x}$. On the other hand, when $b<1, \hat{x}$ is a stable equilibrium.

\section{Theorem 1.}

Fix $t \geq 0$. Let $x(t) \in(0,1)$.

\section{If $b>1$,}
(a) if $x(t)>\hat{x}$, then $x(t+1)>x(t)$, and $x(t) \rightarrow 1$ as $t \rightarrow \infty$
(b) if $x(t)<\hat{x}$, then $x(t+1)<x(t)$, and $x(t) \rightarrow 0$ as $t \rightarrow \infty$;
(c) if $x(t)=\hat{x}$, then for all $t^{\prime}>t, x\left(t^{\prime}\right)=\hat{x}$.

2. If $b<1$,
(a) if $x(t)>\hat{x}$, then $x(t+1)<x(t)$;
(b) if $x(t)<\hat{x}$, then $x(t+1)>x(t)$;
(c) $x(t) \rightarrow \hat{x}$ as $t \rightarrow \infty$.

The opinion $x(t)$ can be interpreted as the individual's degree of support for the extreme represented by 1 . So, the above theorem shows that when the individual is sufficiently biased (i.e., $b>1$ ), exposure to the environment pushes him away from the threshold $\hat{x}$ (unless $x(0)=\hat{x}$ ), and he holds an extreme opinion $(x(t)=0$ or $x(t)=1)$ in equilibrium. Thus, $\hat{x}$ is an unstable equilibrium. This mathematically captures the biased assimilation behavior observed empirically. On the other hand, if the individual has low bias (i.e., $b<1$ ), then he gravitates toward the polarization threshold $\hat{x}$ over time. Thus, $\hat{x}$ is a stable equilibrium in this case. The behavior of the individual when $b=1$ is a limiting case of the two cases proved in the theorem; as $b \rightarrow 1, \lim _{t \rightarrow \infty} x(t)=\hat{x}$, but $\hat{x}$ goes to $1, \frac{1}{2}$, or 0 depending on whether $s$ is greater than, equal to, or less than $\frac{1}{2}$. When the individual is connected to other individuals in a social network, we show below that the biased opinion formation process produces polarization even when $b=1$.

\section{DeGroot's Process Is Not Polarizing}

It is easy to see that if DeGroot's process was asynchronous, i.e., individuals update their opinions one at a time, each opinion update can only lower the NDI. However, here we show that each opinion update can only lower the NDI even when individuals update opinions simultaneously. As a result, the repeated averaging process is depolarizing.

Theorem 2. Consider an arbitrary connected, weighted, undirected graph $G=(V, E, w)$. Let $\mathbf{x} \in[0,1]^{n}$ be an arbitrary vector of opinions of nodes in $G$ at time $t \geq 0$. Assume that for all $i \in V, b_{i}=0$. Then, $\eta(G, \mathbf{x}(t+1)) \leq \eta(G, \mathbf{x}(t))$; i.e., the network disagreement index at time $t+1$ is no more than that at time $t$.

Our result holds for arbitrary weights $w_{i j}$ and an arbitrary vector of opinions $\mathbf{x} \in[0,1]^{n}$, i.e., when the underlying network is arbitrarily homophilous. Moreover, it holds for a number of variants of DeGroot's model that have been proposed to explain the empirically observed lack of consensus on many issues. We defer that discussion to a later section of the paper.

\section{Polarization Due to Biased Assimilation}

In this section we illustrate using a simple model of networks with homophily that the biased opinion formation process may result in polarization, persistent disagreement, or consensus depending on how biased the individuals are. We model homophilous networks using a deterministic variant of multitype random networks (33). Multitype random networks are a generalization of Erdös-Rényi random graphs. Nodes in $V$ are partitioned into types, say, $\tau_{1}, \tau_{2}, \ldots, \tau_{k}$. The network is parameterized by a vector $\left(n_{1}, \ldots, n_{k}\right)$ where $n_{i}$ is the number of nodes of type $\tau_{i}$, and a symmetric matrix $P \in[0,1]^{k \times k}$, where $P_{i j}$ is the probability that there exists an undirected edge between a node of type $\tau_{i}$ and another of type $\tau_{j}$. The class of multitype random networks where $P_{i i}>P_{i j}$ for all $i, j$ is called the islands model and is used to model homophily (because an individual is more likely to be connected with individuals of the same type). We analyze the biased opinion formation process over a deterministic variant of the islands model, which we call a two-island network.

Definition 4. Given integers $n_{1}, n_{2} \geq 0$ and real numbers $p_{s}, p_{d} \in(0,1)$, $a\left(n_{1}, n_{2}, p_{s}, p_{d}\right)$-two-island network is a weighted undirected graph $G=\left(V_{1}, V_{2}, E, w\right)$, where

- $\left|V_{1}\right|=n_{1},\left|V_{2}\right|=n_{2}$, and $V_{1} \cap V_{2}=\varnothing$.

- Each node $i \in V_{1}$ has $n_{1} p_{s}$ neighbors in $V_{1}$ and $n_{2} p_{d}$ neighbors in $V_{2}$.

- $\quad$ Each node $i \in V_{2}$ has $n_{2} p_{s}$ neighbors in $V_{2}$ and $n_{1} p_{d}$ neighbors in $V_{1}{ }^{\dagger}$

- $p_{s}>p_{d}$.

We define the degree of homophily as follows.

Definition 5. Let $G=\left(V_{1}, V_{2}, E, w\right)$ be a $\left(n_{1}, n_{2}, p_{s}, p_{d}\right)$-two-island network. Then the degree of homophily in $G, h_{G}$, is defined to be the ratio $p_{s} / p_{d}$.

${ }^{\dagger}$ For clarity of exposition, we assume that the quantities $n_{1} p_{s}, n_{2} p_{s}, n_{1} p_{d}$ and $n_{2} p_{d}$ are all integers.

(1)

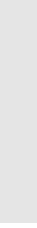


Informally, a high value of $h_{G}$ implies that nodes in $V$ are much more likely to form edges to other nodes of their own type, thereby exhibiting a high degree of homophily.

Theorem 3. Let $G=\left(V_{1}, V_{2}, E, w\right)$ be a $\left(n, n, p_{s}, p_{d}\right)$-two-island network. For all $i \in V=V_{1} \cup V_{2}$, let $w_{i i}=0$. For all $(i, j) \in E$, let $w_{i j}=1$. Assume for all $i \in V_{1}, x_{i}(0)=x_{0}$, where $\frac{1}{2}<x_{0}<1$. Assume for all $i \in V_{2}, x_{i}(0)=1-x_{0}$. Assume for all $i \in V$, the bias parameter $b_{i}=b>0$. Then,

1. (Polarization) If $b \geq 1, \forall i \in V_{1}, \lim _{t \rightarrow \infty} x_{i}(t)=1$, and $\forall i \in V_{2}$, $\lim _{t \rightarrow \infty} x_{i}(t)=0$.

2. (Persistent disagreement) If $1>b \geq \frac{2}{h_{G}+1}$, then there exists $a$ unique $\hat{x} \in\left(\frac{1}{2}, 1\right)$ such that $\forall i \in V_{1}, \lim _{t \rightarrow \infty} x_{i}(t)=\hat{x}$, and $\forall i \in$ $V_{2}, \lim _{t \rightarrow \infty} x_{i}(t)=1-\hat{x}$.

3. (Consensus) If $b<\frac{2}{h_{G}+1}$, then for all $i \in V, \lim _{t \rightarrow \infty} x_{i}(t)=\frac{1}{2}$.

Let us analyze the implications of this theorem. Let $\eta_{\infty}$ be the NDI at equilibrium; i.e., $\eta(G, \mathbf{x}(t)) \rightarrow \eta_{\infty}$ as $t \rightarrow \infty$. Then, the above result implies that when $b \geq 1, \eta_{\infty}>\eta(G, \mathrm{x}(0))$; i.e., the biased opinion formation process is polarizing. On the other hand, when individuals are moderately biased [i.e., $\left.1>b \geq 2 /\left(h_{G}+1\right)\right], \eta_{\infty}>$ $\eta(G, \mathbf{x}(0))$ if and only if $\boldsymbol{x}_{0}<\hat{\boldsymbol{x}}$; so the opinion formation process may not be polarizing, but it does not produce consensus either. Finally, when individuals have low bias [i.e., $\left.b<2 /\left(h_{G}+1\right)\right], \eta_{\infty}=0$. So, the opinion formation process is depolarizing. This illustrates the importance of the bias parameter in causing polarization. Also, observe that $b=1$ corresponds to the urn dynamic described earlier; hence the above result shows that that the urn dynamic causes polarization for an arbitrarily small degree of homophily.

Nonhomogeneous Opinions. Theorem 3 holds in the restrictive setting where initial opinions in each island are homogeneous. However, the biased opinion formation process produces polarization even when initial opinions in each island are not homogeneous. If $b \geq 1$, and the initial opinions of individuals in the two islands are sufficiently far apart relative to the degree of homophily $h_{G}$, the equilibrium opinions of individuals in $V_{1}$ go to 1 and those in $V_{2}$ go to 0 (SI Appendix, Theorem 4.1). Admittedly, in this case, the NDI in equilibrium might be lower than the initial NDI depending on the initial distribution of opinions. However, let us consider another natural measure of opinion divergence, namely, the global disagreement index (GDI).

Definition 6. Global Disagreement Index. Given a vector of opinions $\mathbf{x} \in[0,1]^{n}$ of individuals in $V$, the global disagreement index $\gamma(\mathbf{x})$ is defined as

$$
\gamma(\mathbf{x}):=\sum_{i<j}\left(x_{i}-x_{j}\right)^{2}
$$

The GDI is maximized when half the individuals have opinion 0 and other half 1 . So, regardless of the initial distribution of opinions, the biased opinion formation process produces polarization even in this case, if opinion divergence is measured using GDI.

\section{Variants of DeGroot's Process}

Our result about DeGroot's process (Theorem 2) in fact holds for a number of variants that are all based on repeated averaging of opinions. Here we discuss some of the variants.

Stubborn Individuals. One variant (34) of DeGroot's model attempts to explain the observed lack of consensus on many issues by allowing some nodes to have an infinite self-weight w_ii. Such nodes are called stubborn individuals. Because our result holds for arbitrary weights, this variant is also depolarizing according to our definition.

Surprising Validators. It has been argued (27) that biased assimilation can be countered by surprising validators: Individuals are more likely to carefully consider disconfirming evidence if it is presented by a source that is otherwise similar to them. An opinion formation process with surprising validators can be viewed as individual $i$ adopting an opinion held by $j$, if $i$ finds $j$ to be similar to him. This process can be interpreted as the following natural urn dynamic: At each time step, $i$ chooses a neighbor $j$ with probability proportional to $w_{i j}$ and inspects a ball chosen uniformly at random from $j$ 's urn. $i$ also inspects a ball uniformly at random from his own urn. If the colors of the two inspected balls match, $i$ inspects another ball chosen uniformly at random from $j$ 's urn, adds a ball of the same color to his urn, and discards a ball chosen uniformly at random from his urn.

Observe that conditioned on the colors of the two inspected balls matching, the probability that $i$ adds a RED ball to his urn is $x_{j}(t)$, which is identical to the corresponding (unconditional) probability in DeGroot's process. In other words, this process is a conditional version of DeGroot's process. Mathematically, the opinion update in this process is given by

$$
x_{i}(t+1):=\frac{w_{i i} x_{i}(t)+\sum_{j \in N(i)} w_{i j} p_{i j}(t) x_{j}(t)}{w_{i i}+\sum_{j \in N(i)} w_{i j} p_{i j}(t)},
$$

where the additional term $p_{i j}(t):=\left(x_{i}(t)\right)^{b_{i}} x_{j}(t)+\left(1-x_{i}(t)\right)^{b_{i}}(1-$ $\left.x_{j}(t)\right)$ corresponds to the probability in the urn model that $i$ finds $j$ to be similar to him. Observe that if we define $w_{i j}^{\prime}(t):=w_{i j} p_{i j}(t)$ [7] is identical to [2], except that the weights may vary with time. Therefore, like DeGroot's process, each update in the opinion formation process with surprising validators can only lower the NDI, regardless of the value of the bias parameter $b_{i}$. This stylized model validates the claim $(27,28)$ that biased assimilation can be countered with surprising validators.

Flocking Model. The flocking model is a well-known model for decentralized consensus (35) based on repeated averaging. Under this model, at each time step $t \geq 0$, an arbitrary set $S(t) \subseteq V$ of individuals simultaneously updates their opinions to be closer to the average opinion of the set.

Definition 7. Flocking Process. Let $\epsilon \in[0,1]$. For $t \geq 0$, let $S(t) \subseteq V$ be an arbitrary set of individuals such that $|S(t)| \geq 2$. Let $s(t):=$ $\frac{1}{|S(t)|} \sum_{i \in S(t)} x_{i}(t)$ be the average opinions of individuals in $S(t)$. Under the flocking process, the opinion of individual $i \in V$ at time $t+1$, $x_{i}(t+1)$, is given by

$$
x_{i}(t+1)=\left\{\begin{array}{cc}
(1-\epsilon) x_{i}(t)+\epsilon S(t), & \text { if } i \in S(t) \\
x_{i}(t), & \text { otherwise. }
\end{array}\right.
$$

Observe that in the flocking process, there is no notion of an underlying network. Therefore, the GDI (Definition 6) is a natural measure of opinion divergence under this process. Next we show that each opinion update in the flocking process can only lower the GDI.

Theorem 4. Let $\mathbf{x} \in[0,1]^{n}$ be an arbitrary vector of opinions of nodes in $V$ at time $t \geq 0$. Let $\mathbf{x}(t+1) \in[0,1]^{n}$ be the vector of opinions at time $t+1$ after one step of the flocking process. Then, $\gamma(\mathbf{x}(t+1)) \leq \gamma(\mathbf{x}(t))$; i.e., the GDI at time $t+1$ is no more than that at time $t$.

A generalization of the GDI is the following: $\sum_{i<j} h\left(\left|x_{i}-x_{j}\right|\right)$, where $h$ is an arbitrary convex function. The flocking process has the property that the vector $\mathbf{x}(t+1)$ is majorized by $\mathbf{x}(t)$. Therefore, as noted in the proof of Theorem 4, each opinion update of the flocking process is depolarizing under this definition or, more generally, when divergence is defined by any symmetric convex function of $\mathbf{x}$.

Observe that it is possible to assign weights $w_{i j}$ such that a single opinion update in DeGroot's process increases the GDI (or any symmetric convex function of $\mathbf{x}$ ) because the latter is independent of the weights. However, DeGroot's process converges to 
consensus under fairly general conditions (18). Thus, under those conditions, DeGroot's process is depolarizing in equilibrium.

\section{Recommender Systems and Polarization}

Recommender systems are widely used on the Internet to present personalized information (e.g., search results, news articles, products) to individuals. This personalization is typically done by algorithms that use an individual's past behavior (e.g., history of browsing and purchases) and that of other individuals that are similar in some way to that individual, to discover items of possible interest to the user. It has been argued (17) that this personalization has an echo-chamber effect where individuals are exposed only to information they agree with, and this ultimately leads to increased polarization. Here we investigate this question: Do recommender systems have a polarizing effect?

We consider the following simple model: Let $G=\left(V_{1}, V_{2}, E\right)$ be an unweighted undirected bipartite graph. Nodes in $V_{1}$ represent individuals. Nodes in $V_{2}$ represent items. The items could be books, webpages, news articles, products, etc. For concreteness, we refer to nodes in $V_{2}$ as books. For a node $i \in V_{1}$ and a node $j \in V_{2}$, an edge $(i, j) \in E$ represents ownership, i.e., individual $i$ owns book $j$. For our purpose, we define a recommender algorithm as below.

Definition 8. $A$ recommender algorithm takes as input a bipartite graph $G=\left(V_{1}, V_{2}, E\right)$ and a node $i \in V_{1}$ and outputs a node $j \in V_{2}$

Thus, given a graph representing which users own which books and a specific user $i$, a recommender algorithm outputs a single book $j$ to be recommended to $i$. We analyze three simple recommender algorithms - SimpleSALSA (Algorithm 1), SimpleICF (Algorithm 2), and SimplePPR (Algorithm 3) - that are similar in spirit to three well-known recommender algorithms from the literature: SALSA (36), Personalized PageRank (29), and itembased collaborative filtering (37), respectively. All three algorithms are based on performing random walks on the graph $G$. Informally speaking, SimpleSALSA and SimpleICF first choose a random item liked by user $i$ and recommend an item similar to that item, whereas SimplePPR recommends the item that is most relevant to user $i$ on the basis of a PageRank-like score.

We assume that $i$ can buy a book only if it is recommended to him. However, he may choose to reject a recommendation, i.e., to not buy a recommended book. Therefore, $i$ buying a book $j$ requires two steps: The recommender algorithm must recommend $j$ to $i$, and then $i$ must accept the recommendation.

Because we are interested in analyzing the polarizing effects of recommender systems, we assume that each book in $V_{2}$ is labeled either "RED" or "BLUE". These labels are purely for the purpose of analysis; the algorithms we study are agnostic to these labels. For each individual $i \in V_{1}$, let $x_{i} \in[0,1]$ be the fraction of RED books owned by $i$ and $1-x_{i}$ be that of BLUE books. Individuals may be biased or unbiased, as we define below.

Definition 9. Consider a book recommended to an individual $i \in V_{1}$. We say that $i$ is unbiased if $i$ accepts the recommendation with the same probability independent of whether the book is RED or $B L U E$. We say that $i$ is biased if

1. $i$ accepts the recommendation of a RED book with probability $x_{i}$ and rejects it with probability $1-x_{i}$ and

2. $i$ accepts the recommendation of a BLUE book with probability $1-x_{i}$ and rejects it with probability $x_{i}$.

Observe that the above definition of an individual $i$ being biased corresponds to the urn dynamic described earlier with $b_{i}=1$. For an individual $i$, the fraction of RED books $i$ owns, $x_{i}$, can

\section{Algorithm 1. SimpleSALSA}

Input: $G=\left(V_{1}, V_{2}, E\right)$, node $i \in V_{1}$.

1: Perform a three-step random walk on $G$ starting at $i$.

2: Let the random walk end at node $j \in V_{2}$.

Output: $j$.
Algorithm 2. SimpleICF

Input: $G=\left(V_{1}, V_{2}, E\right)$, node $i \in V_{1}$.

Parameter: A large positive integer $T$.

1: Choose a neighbor $k$ of $i$ uniformly at random.

2: Perform $T$ two-step random walks on $G$ starting at $k$.

3: For each node $j \in V_{2}$, let count (j) be the number of random walks that end at node $j$.

4: Let $j^{*}:=\arg \max _{j}$ count (j).

Output: $j^{*}$.

be viewed as $i$ 's opinion in the interval $[0,1]$, and so a recommender algorithm can be viewed as an opinion formation process. The opinion $x_{i}$ remains unchanged if $i$ rejects a recommendation. However, if $i$ accepts a recommendation, $x_{i}$ increases or decreases depending on whether the recommended book was RED or BLUE. Thus, we are interested in the probability that a recommendation was for a RED (or BLUE) book given that $i$ accepted the recommendation. The above probability determines whether a recommender algorithm is polarizing or not.

Definition 10. Consider a recommender algorithm and an individual $i \in V_{1}$ that accepts the algorithm's recommendation. The algorithm is polarizing with respect to $i$ if

1. when $x_{i}>\frac{1}{2}$, the probability that the recommended book was RED is greater than $x_{i}$, and

2. when $x_{i}<\frac{1}{2}$, the probability that the recommended book was RED is less than $x_{i}$.

Informally speaking, a recommender algorithm is polarizing if it makes a "RED individual" more RED and a "BLUE individual" more BLUE. To analyze the recommender algorithms, we assume a generative model for $G$, which we describe next.

Generative Model for $\boldsymbol{G}$. Let the number of individuals, $\left|V_{1}\right|=m>0$. Let the number of books, $\left|V_{2}\right|=2 n$, with $n>0$ books of each color. We assume that $m=f(n)$; and $\lim _{n \rightarrow \infty} f(n)=\infty$. For individual $i \in V_{1}$, we draw $x_{i}$ independently from a distribution over $[0,1]$ with a probability density function $g(\cdot)$. We assume that $g$ is symmetric about $\frac{1}{2}$; i.e., for all $y \in[0,1], g(y)=g(1-y)$. This implies that for all $i \in V_{1}, \mathbb{E}\left[x_{i}\right]=\frac{1}{2}$. We assume that the variance of the distribution is strictly positive; i.e., $\operatorname{Var}\left(x_{i}\right)>0$. For an individual $i$ and a RED book $j$, there exists an edge $(i, j) \in E$ independently with probability $\frac{x_{i} k}{n}$, where $0<k<n$. For an individual $i$ and a BLUE book $j$, there exists an edge $(i, j) \in E$ independently with probability $\frac{\left(1-x_{i}\right) k}{n}$. So, in expectation, each individual $i$ owns $k$ books, and $x_{i}$ fraction of them are RED.

For two books $j, j^{\prime} \in V_{2}$, let $M_{i j^{\prime}}:=\left|N(j) \cap N\left(j^{\prime}\right)\right|$ be the number of individuals in $V_{1}$ that are neighbors of both $j$ and $j^{\prime}$ in $G$. For any two nodes $i, j \in V$, let $\mathbb{P}[i \stackrel{\ell}{\longrightarrow} j]$ be the probability that a $\ell$-step random walk over $G$ starting at $i$ ends at $j$. For a node $i \in V_{1}$ and a node $j \in V_{2}$, let $Z_{i j}$ be the indicator variable for edge $(i, j)$; i.e., $Z_{i j}=1$ if $(i, j) \in E$, and $Z_{i j}=0$ otherwise.

Analysis. Next we prove our results about the polarizing effects of each of the three algorithms. Our results rely on a technical lemma, stated in SI Appendix, Lemma 6.1, which invokes the Strong law of large numbers to show that random quantities such as the number of neighbors of a user $i$ or of a book $j$ in the graph $G$ all take their expected values with probability 1 as $n \rightarrow \infty$. First we show that SimplePPR (Algorithm 3) is polarizing with respect to $i$ even if $i$ is unbiased.

Theorem 5. Fix a user $i \in V_{1}$. In the limit as $n \rightarrow \infty$ and as $T \rightarrow \infty$, SimplePPR is polarizing with respect to $i$.

Next we show that SimpleSALSA (Algorithm 1) and SimpleICF (Algorithm 2) are polarizing only if $i$ is biased.

Theorem 6. Fix a user $i \in V_{1}$. In the limit as $n \rightarrow \infty$, 


\section{Algorithm 3. SimplePPR}

Input: $G=\left(V_{1}, V_{2}, E\right)$, node $i \in V_{1}$.

Parameter: A large positive integer $T$.

1: Perform $T$ three-step random walks on $G$ starting at $i$.

2: For each node $j \in V_{2}$, let count (j) be the number of random walks that end at node $j$.

3: Let $j^{*}:=\arg \max _{j}$ count (j).

Output: $j^{*}$.

1. SimpleSALSA is polarizing with respect to $i$ if and only if $i$ is biased.

2. In the limit as $T \rightarrow \infty$, SimpleICF is polarizing with respect to $i$ if and only if $i$ is biased.

Both SimpleICF and SimpleSALSA first choose a random book owned by $i$ : They choose a RED book with probability $x_{i}$ and a BLUE book with probability $1-x_{i}$. This initial random choice ensures that recommendations are sufficiently diverse; i.e., the book eventually recommended by these algorithms is RED with probability at most $x_{i}$ when $x_{i}>\frac{1}{2}$. Recall that our definition of a biased individual in this section corresponds to $b=1$. However, as we point out in the proof of Theorem 6 , both algorithms are polarizing for all $b \geq 1$.

By contrast, the reason why SimplePPR is always polarizing is because the large number of three-step random walks serves to amplify user $i$ 's initial preference: If $x_{i}>\frac{1}{2}$, SimplePPR recommends a RED book is probability 1 , and vice versa. Consequently, as we point out in the proof, Theorem 5 holds for all $b \geq 0$.

Analyzing the polarizing effect of recommender algorithms under a setting where the graph $G$ evolves over time is an interesting question that we leave for future work.

\section{Concluding Remarks}

In this paper we attempted to explain polarization in society through a model of opinion formation. We showed that DeGroot-like

1. Poole KT, Rosenthal H (1984) The polarization of american politics. J Polit 46(4): 1061-1079.

2. Poole KT, Rosenthal H (1991) Patterns of congressional voting. Am J Po/ Sci 35(1):228-278

3. Hill S (2009) Divided we stand: The polarization of American Politics. Natl Civ Rev 94(4):3-14.

4. McCarty N, Poole KT, Rosenthal H (2006) Polarized America: The Dance of Ideology and Unequal Riches (MIT Press, Cambridge, MA).

5. Schelling TC (1971) Dynamic models of segregation. J Math Sociol 1:143-186.

6. Bruch EE, Mare RD (2006) Neighborhood choice and neighborhood change. Am J Sociol 112(3):667-709.

7. Brandt C, Immorlica N, Kamath G, Kleinberg R (2012) An analysis of one-dimensiona Schelling segregation. Proc 44th symposium on Theory of Computing, STOC '12, eds Karloff HJ, Pitassi T (Association for Computing Machinery, New York), pp 789-804.

8. Carter B (April 27, 2009) With rivals ahead, doubts for CNN's middle road. NY Times, Business Section, $\mathrm{p}$ B1.

9. Carter B (March 29, 2010) CNN fails to stop fall in ratings. NY Times, Business Section, p B1.

10. Adamic L, Glance N (2005) The political blogosphere and the 2004 U.S. election: Divided they blog. In LinkKDD 05: Proc Third International Workshop on Link Discovery, eds Adibi J, Grobelnik M, Mladenic D, Pantel P (Association for Computing Machinery, New York), pp 36-43.

11. Hargittai E, Gallo J (2007) Cross-ideological discussions among conservative and liberal bloggers. Public Choice 134:67-86.

12. Gilbert E, Bergstrom T, Karahalios K (2009) . Blogs are echo chambers: Blogs are echo chambers.Proceedings of the Hawaii International Conference on System Sciences, ed Sprague RH, Jr. (IEEE Computer Society, Wasington DC), pp 1-10.

13. Lawrence E, Sides J, Farrell H (2010) Self-segregation or deliberation? Blog readership, participation, and polarization in American politics. Perspect Polit 8(1):141-157.

14. Wolfe A (1999) One Nation, After All: What Americans Really Think About God, Country, Family, Racism, Welfare, Immigration, Homosexuality, Work, The Right, The Left and Each Other (Penguin Group, New York).

15. Fiorina MP, Abrams SJ, Pope JC (2005) Culture War? The Myth of a Polarized America (Pearson Education, New York).

16. Baron RS, et al. (1996) Social corroboration and opinion extremity. J Exp Soc Psycho 32(6):537-560.

17. Sunstein CR (2002) Republic.com (Princeton Univ Press, Princeton, NJ).

18. DeGroot MH (1974) Reaching a consensus. J Am Stat Assoc 69(345):118-121.

19. Friedkin NE, Johnsen EC (1990) Social influence and opinions. J Math Sociol 15(3-4): 193-206. repeated averaging processes can never be polarizing, even if individuals are arbitrarily homophilous. We generalized DeGroot's repeated averaging model to account for biased assimilation. We showed that in a two-island network, our biased opinion formation process results in polarization when individuals are sufficiently biased. In other words, homophily alone, without biased assimilation, is not sufficient to polarize society. We also used biased assimilation to provide insight into the polarizing effects of three recommender algorithms. We showed that SimplePPR, which recommends the item that is most relevant to a user on the basis of a PageRank-like (29) score, is always polarizing. The other two algorithms, which first choose a random item liked by the user and recommend an item similar to that item, are polarizing only if individuals are biased.

Our analysis raises a number of questions that we view as promising directions for further research. For example, are recommender algorithms that produce more relevant recommendations necessarily more polarizing? For certain applications (e.g. ecommerce), polarizing effects may not be an overriding concern. On the other hand, for online social systems designed expressly to facilitate collective decision making regarding complex societal issues, polarization might be a dominant concern. In the case of recommender algorithms for news articles, blogs, etc., there may well be a trade-off between relevance and polarizing effects. An understanding of polarization, its causes, and associated trade-offs is important for designing Internet-based socioeconomic systems.

As a final note, complete proofs of all theorems are presented in SI Appendix.*

ACKNOWLEDGMENTS. This research was supported in part by Nationa Science Foundation (NSF) Grants 0904325 and 0947670. D.T.L. was supported in part by a NSF Graduate Research Fellowship under Grant DGE-1147470.

*A preliminary version of this paper was presented at the Eighth Workshop on Internet and Network Economics held at the University of Liverpool (Liverpool, UK), December 9-12, 2012

20. Krause U (2000) A discrete nonlinear and non-autonomous model of consensus for mation. Communications in Difference Equations, eds Elaydi S, Ladas G, Popenda J Rakowski J (Gordon and Breach Science Publishers, Amsterdam), pp 227-236.

21. Acemoglu D, Como G, Fagnani F, Ozdaglar AE (2010) Opinion fluctuations and disagreement in social networks. arXiv:1009.2653.

22. Bindel D, Kleinberg JM, Oren S (2011) How bad is forming your own opinion? Proc IEEE Symposium on Foundations of Computer Science, ed Ostrovsky R (Institute of Electrical and Electronic Engineers Computer Society, Washington DC), pp 57-66.

23. Lord CG, Ross L, Lepper MR (1979) Biased assimilation and attitude polarization: The effects of prior theories on subsequently considered evidence. J Pers Soc Psychol 37(11):2098-2109.

24. Miller AG, McHoskey JW, Bane CM, Dowd TG (1993) The attitude polarization phe nomenon: Role of response measure, attitude extremity, and behavioral consequences of reported attitude change. J Pers Soc Psychol 64(4):561-574.

25. Munro GD, et al. (2002) Biased assimilation of sociopolitical arguments: Evaluating the 1996 U.S. presidential debate. Basic App/ Soc Psych 24(1):15-26.

26. Taber CS, Lodge M (2006) Motivated skepticism in the evaluation of political beliefs. Am J Pol Sci 50(3):755-769.

27. Sunstein C (September 18, 2012) Breaking up the echo. NY Times, Op-Ed Section, p A25.

28. Centola D (2011) An experimental study of homophily in the adoption of health behavior. Science 334(6060):1269-1272.

29. Page L, Brin S, Motwani R, Winograd T (1999) The PageRank Citation Ranking: Bringing Order to the Web, Technical Report (Stanford InfoLab, Stanford, CA).

30. Heider F (1946) Attitudes and cognitive organization. J Psychol 21(1):107-112.

31. Festinger L (1957) A Theory of Cognitive Dissonance (Stanford Univ Press, Stanford, CA).

32. Baldassarri D, Gelman A (2008) Partisans without constraint: Political polarization and trends in American public opinion. Am J Sociol 114(2):408-446.

33. Golub B, Jackson MO (2011) How homophily affects the speed of learning and best response dynamics. $Q$ J Econ 127(3):1287-1338.

34. Yildiz E, Acemoglu D, Ozdaglar AE, Saberi A, Scaglione A (2011) Discrete opinion dynamics with stubborn agents. SSRN eLibrary, in press.

35. Tsitsiklis JN (1984) Problems in decentralized decision making and computation. PhD thesis (Department of Electrical Engineering and Computer Science, Massachusetts Institute of Technology, Cambridge, MA).

36. Lempel R, Moran S (2001) Salsa: The stochastic approach for link-structure analysis. ACM Trans Inf Syst 19(2):131-160.

37. Linden G, Smith B, York J (2003) Amazon.com recommendations: Item-to-item collaborative filtering. Internet Computing, IEEE 7(1)76-80. 